\title{
Energy Aware Routing Using Improved LEACH Protocol
}

\author{
Mehwish Mumtaz, Aleena Mumtaz, Muhammad N. Khan* \\ Department of Electrical Engineering, The University of Lahore, Pakistan
}

Copyright (C) 2015 by authors, all rights reserved. Authors agree that this article remains permanently open access under the terms of the Creative Commons Attribution License 4.0 International License

\begin{abstract}
In this research paper, we propose an energy efficient routing protocol low energy adaptive clustering hierarchy (LEACH) for wireless sensor networks. There are many sensing nodes and sink nodes in wireless sensor network system. Routing protocol and its improvement is a major issue in wireless sensor networks. Our proposed protocol is the modification of LEACH. The main idea is to increase the network life and maximizes the throughput. In this research paper, residual energy is to be considered for the selection of cluster head from various nodes. Time division multiple access (TDMA) technique is used for the communication between common nodes and cluster head. Cluster head $(\mathrm{CH})$ chooses code to be used in its cluster and code division multiple access (CDMA) technique is used between clusters. The comparison between improved versions of LEACH protocol shows that multi-hop LEACH shows significant improvement than energy LEACH (E-LEACH).
\end{abstract}

Keywords Wireless Sensor Networks (WSN), Cluster Head (CH), E-LEACH (Energy Low-energy Adaptive Clustering Hierarchy), MR-LEACH (Multi-hop Routing LEACH)

\section{Introduction}

Wireless sensor network (WSN) consists of sensing nodes, base station (BS) and common nodes. Use of efficient energy is the main purpose of sensing nodes in WSN. The main objective of sensing nodes is to sense and aggregate the data towards BS. Comparing with non-cluster algorithm, cluster based algorithm are best with respect to the energy consumption [1-3]. In clustering algorithm LEACH is the best because it is more efficient. In LEACH, network is divided into cluster and run time is divided into rounds. All common nodes have equal probability to be selected as cluster head $(\mathrm{CH})$, it makes balance the distribution of energy such as lifetime of network increases. We improve this protocol by multi-hop routing, data is routed so that routes have minim cost. In $\mathrm{M}$-LEACH there is unequal clustering.
In $\mathrm{M}-\mathrm{LEACH}$, nodes with maximum energy perform advertisement, showing the willingness of becoming $\mathrm{CH}[2$, 4]. $\mathrm{CH}$ selection depends on the receive strength of the signal. When $\mathrm{CH}$ is established, communication starts. Each node choses its $\mathrm{CH}$ in order to arrive at $\mathrm{BS}$. This procedure is continuing until a change is formed to reach the BS. TDMA schedule is used for scheduling network level. In our paper we enhance the LEACH protocol to E-LEACH and modified multi-hop LEACH. We develop comparisons between the traditional LEACH with modified protocols.

\section{Leach Protocol}

The main idea of LEACH is that whole network is divided into many clusters. There is an equal opportunity of each node to become a $\mathrm{CH}$. The selection criterion for the nodes to become $\mathrm{CH}$ first time is random. The networks lifetime can be prolonged. LEACH also provides the concept of rounds. In LEACH protocol each round contains two states, set up and steady state phases. In cluster set up phase formation of clusters occurs in a self-adaptive mode [5-8]. In steady state, transformation of data takes place. The second state takes more time as compared to the first state, because it saves the protocol payload.

At initial state each sensor node wants to become a cluster head on the basis of suggested percentage of cluster heads in the network $[4,9]$. The node decides by selecting a random number between 0 and 1 . If this random number is less than $\mathrm{T}(\mathrm{n})$, the node become a cluster head for this round. The threshold is given as:

$$
\begin{gathered}
T(n)=\frac{p}{1-p \times\left(r \bmod p^{-1}\right)^{\prime}} \forall n \in G \\
T(n)=0, \forall n \in G
\end{gathered}
$$

where in (1), p is the percentage of the required cluster heads, $r$ is the current round and $\mathrm{G}$ is the nodes that are not $\mathrm{CHs}$ during the last $1 / \mathrm{p}$ rounds. Each node will be a cluster head at some point within $1 / \mathrm{p}$ rounds. Using (1), every node becomes $\mathrm{CH}$ at any point within $1 / \mathrm{p}$ round. All those nodes that have been $\mathrm{CHs}$ during previous rounds cannot become CHs for $1 / \mathrm{p}-1$ round. A TDMA schedule is built up by cluster 
head node sets up and transmits this schedule to nodes in $\mathrm{CH}$ and the set up phase is completed. The steady state phase is phase in which data is transmit among the cluster after the establishment of cluster heads in setup phase and when data is need to transmit, they will send an idle slots TDMA table to the cluster head.

\section{Proposed Algorithm}

In our proposed algorithm we present LEACH, energy -LEACH and multi-hop LEACH using improved hierarchy. In this improvement we suggest clustering concept and dividing into setup and data transfer phase. As discussed in Section 2, improved formula for the selection of $\mathrm{CHs}$ and then collect the aggregated data from common nodes toward BS. For such task, common nodes consume less energy than the cluster head. Some main issues in LEAH are as follows;

1) There is random selection of $\mathrm{CHs}$ in the LEACH because of the equal probability in the selection of $\mathrm{CHs}$, some short coming occur in LEACH protocol [5]. There are many rounds in LEACH and there is equal probability of node with less energy and with more residual energy to become a cluster head. In case, a node having less energy become as cluster head, its energy will be consume and thus quickly died. Hence, lifetime of the whole network will be effected and shortened.

2) In LEACH protocol clusters are randomly formed and unequal distribution of clusters occur. This leads to the fact that divided clusters are not best. Some cluster heads will be in center of cluster has more energy than the others in edge of clusters. To overcome these issues our proposed protocols are:

- Energy LEACH protocol

- Multi hop LEACH protocol

\section{Energy Leach Protocol}

In E-LEACH selection criteria of CHs is improved. The two main categories of routing and transfer of information in network are discussed here. E-LEACH will select those cluster head which have none uniform initial energy level among other sensing node [10-15]. It can be determine that $\mathrm{CHs}$ which are required has to scale the total sensing node in order to minimize energy distribution in network. All the remaining aspects other than above two of E-LEACH are equal to traditional LEACH protocol as is shown in Fig. 1.

In E-LEACH after first round either a node will become a $\mathrm{CH}$ on not depend upon residual energy. In E-LEACH on the basis of probability each node turn as $\mathrm{CH}$.i.e., there is random selection of $\mathrm{CHs}$ initially. In second round residual energy of each node is differ from each other. Thus node have more energy will become $\mathrm{CH}$ rather than less energy node.

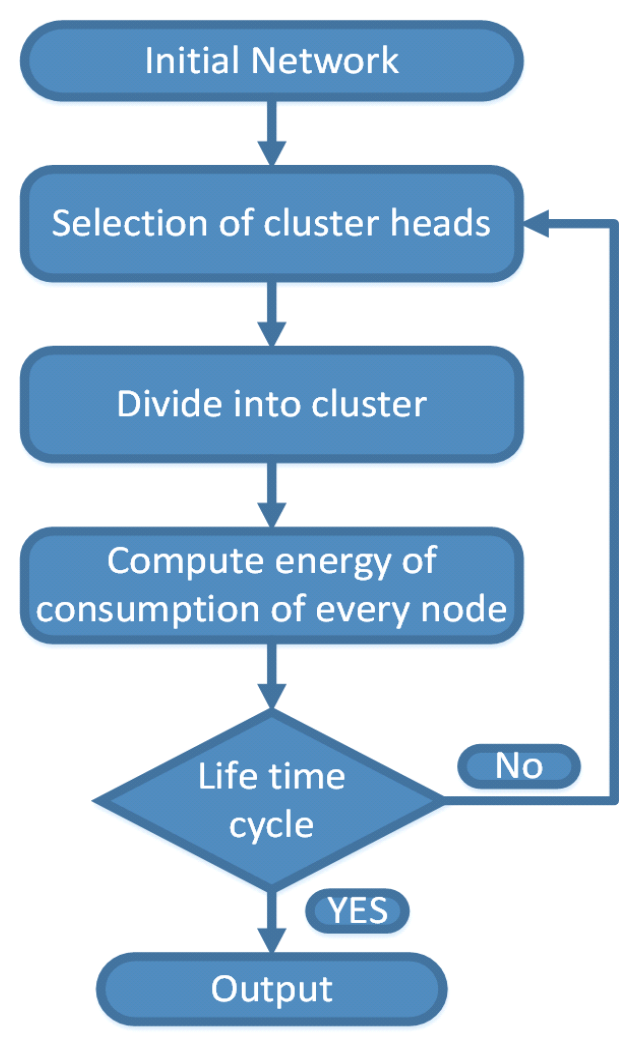

Figure 1. Flow chart of Energy LEACH protocol

\section{Multi-hop Routing Leach Protocol}

M-LEACH is same as the traditional LEACH protocol, the only difference is that in M-LEACH communication occurs from single hop to multi hop between $\mathrm{CH}$ and common node. The basic idea behind the M-LEACH is to reduce the energy consumption between cluster head and BS. On the basis of TDMA, every $\mathrm{CH}$ has its own schedule for all nodes [3].

In M-LEACH CHs collect the data in order to route data to BS $[7,16]$. This process yield longer network life. This process of multi-hope clustering is provided in Table-1. In MR-LEACH approach variable number of intermediate nodes will forward data to the base station, depending on the location of the sensed data.

Table 1. MR Leach Protocol Algorithm

\begin{tabular}{|c|c|}
\hline MR-LEACH & Description \\
\hline Step1 & In the first hop, each node send data towards CH \\
\hline Step2 & $\begin{array}{c}\text { An efficient energy node is selected to forward packet } \\
\text { from previous node. }\end{array}$ \\
\hline Step3 & After that nearest node is selected for data \\
\hline Step4 & In the last hop data is send to selected node \\
\hline
\end{tabular}




\section{Simulation Results}

The sensor nodes are randomly distributed in a square region. After the normal nodes are deployed, the base stations and nodes are fixed and the cluster-heads rotate randomly. The energy of node may be heterogeneous, but the energy restriction cannot be added, the energy and the storage capacity of the base station are not limited [8]. Nodes can communicate not only with each other in the network, but also with the base station directly. Node can adjust the wireless transmission power according to the distance. To evaluate the algorithm performance, we simulate both E-LEACH and Multi-hop LEACH by MATLAB.

The simulation parameters are provided in Tab-2. Figure 2 shows the performance of residual energy of LEACH protocol, our E-LEACH and MR-LEACH.
Table 2. Simulation parameters

\begin{tabular}{|c|c|}
\hline Simulation Parameters & Values \\
\hline Distance between member node and CHs & $\mathrm{R}$ \\
\hline Average distance between two successive CHs & $1.5 \mathrm{R}$ \\
\hline Total sensing field & $100 \mathrm{~m} \times 100 \mathrm{~m}$ \\
\hline Average distance between node and CHs & $135 \mathrm{~m}$ \\
\hline Total number of layers in network & $\mathrm{N}$ \\
\hline When message is relayed using clustering hierarchy & {$[\mathrm{N} / 2]$} \\
\hline $\begin{array}{c}\text { In LEACH average distance between cluster head } \\
\text { and BS }=\end{array}$ & $110 \mathrm{~m}$ \\
\hline Data packet size $=K$ & $200 \mathrm{bits}$ \\
\hline $\begin{array}{c}\text { The energy being dissipated to run the transmitter: } \\
E_{\text {elec }}\end{array}$ & $50 \mathrm{~nJ} /$ bit \\
\hline $\begin{array}{c}\text { The energy dissipation of the transmission } \\
\text { amplifier: } \boldsymbol{E}_{a m p}\end{array}$ & $100 \mathrm{pJ} /$ bit \\
\hline
\end{tabular}

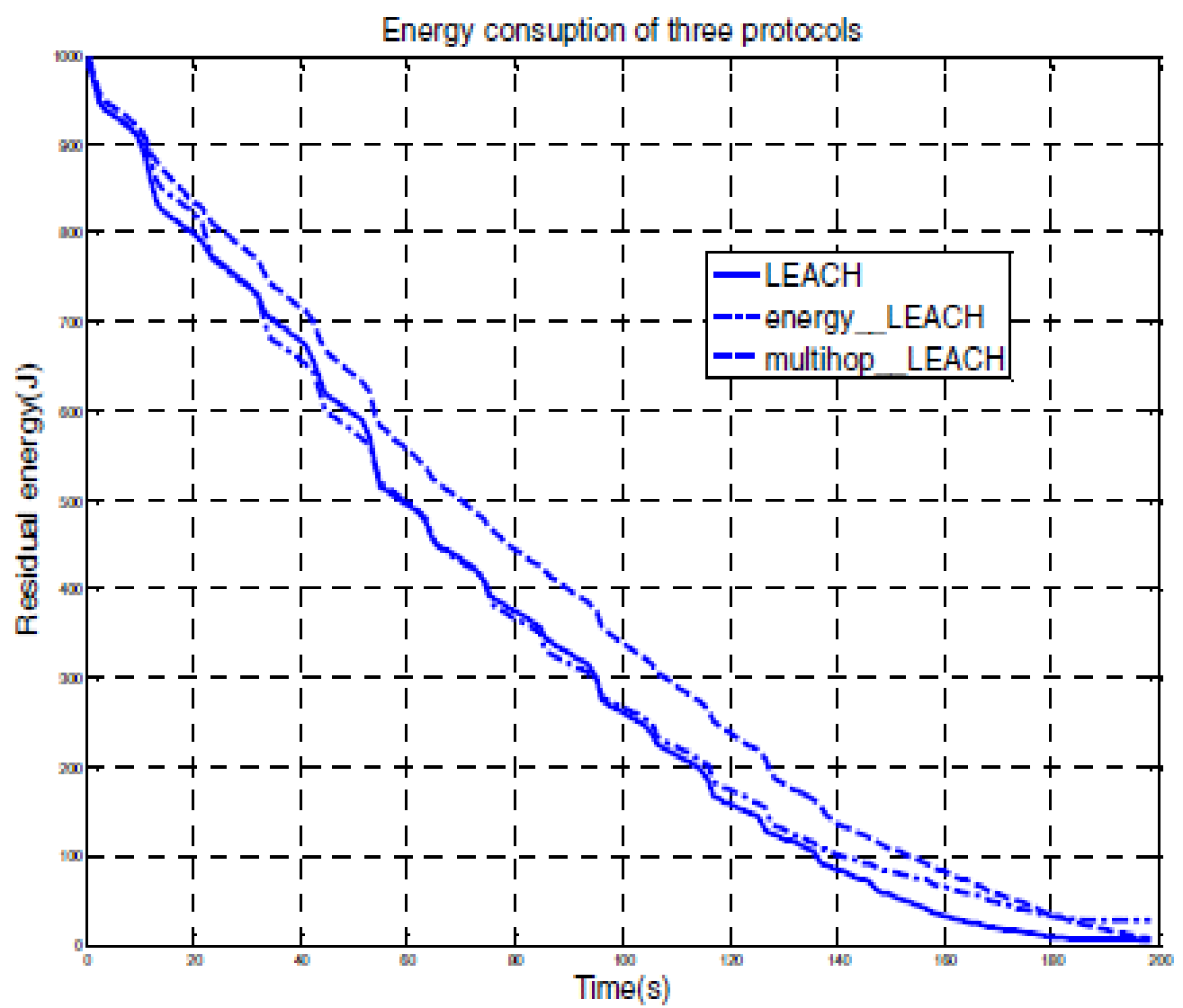

Figure 2. Residual energy versus time. 
In Fig. 3, Multi-hop-LEACH protocol has more residual energy than LEACH and energy-LEACH protocols. Energy-LEACH protocol has the same residual energy as LEACH protocol in the beginning.

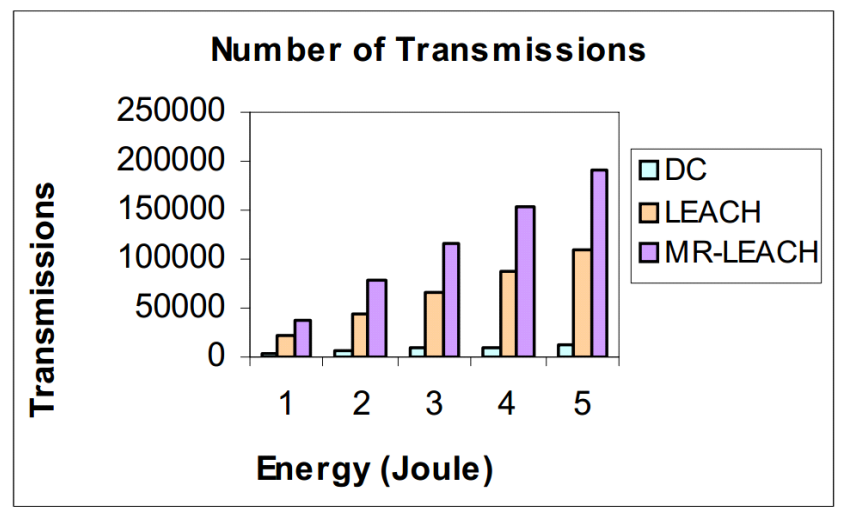

Figure 3. Histogram of Transmissions

\section{Conclusions}

In this paper, energy aware routing using improved LEACH protocol shows that M-LEACH protocol is better than the traditional LEACH and E-LEACH. We solve the issue of selecting $\mathrm{CH}$, by equal distribution of each node becoming cluster head. The energy consumption issue is also solved by above two modified protocols. In our simulation result, we compare the LEACH with improved LEACH and we observe that the improved LEACH protocol achieves the balance of network's flow. In this research paper, energy efficiency is achieved by minimizing the packet size in multi-level data aggregation among cluster head and particular selection of CHs among nodes of cluster.

\section{REFERENCES}

[1] Poonam Tyagi, Ravinder Prakash Gupta, Rakesh Kumar Gill,"Comparative Analysis of Cluster Based Routing Protocols used in Heterogeneous Wireless Sensor Network", International Journal of Soft Computing and Engineering (IJSCE) ISSN: 2231-2307, vol. 1, no. 5, pp. 2231-2307, Nov. 2014

[2] Xu, G. Yang, Z.-Yu. Chen, L. Chen, and Z. Yang, "Performance analysis of data aggregation algorithms in wireless sensor networks," in Proceedings of the International Conference on Electrical and Control Engineering (ICECE '11), September 2013.

[3] Akyildiz and M. C. Vuran, Wireless Sensor Networks, University Publishers, Tsinghua, China, 2011
[4] Nam and H. Min, "An Energy-Efficient Clustering Using a Round-Robin Method in a Wireless Sensor Network".5th ACIS International Conference on Software Engineering Research, Management \& Applications, 2013, pp. 54-60.

[5] N.M.A. Latiff, C.C. Tsimenidis, and B.S. Sharif, "Performance Comparison of Optimization Algorithm for Clustering in Wireless Sensor Networks," IEEE International Conference on Mobile Adhoc and Sensor Systems, 2010, pp. $1-4$.

[6] Z. Zhang and X. Zhang., "Research of Improved Clustering Routing Algorithm Based on Load Balance in Wireless Sensor Networks", IET International Communication Conference on Wireless Mobile and Computing, 2009, pp. 661-664.

[7] W.R. Heinzelman, A. Chandrakasan, and H. Balakrishnan, "Energy-efficient communication protocol for wireless microsensor networks," Proceedings of the 33rd Hawaii International Conference on System Sciences, 2009, pp. 1-10.

[8] W.R. Heinzelman, A. Chandrakasan, and H. Balakrishnan,"An Applications-Specific Protocol Architecture for Wireless Microsensor Networks," IEEE Transactions on Wireless Communications, 2008, vol. 1, no. 4, 2008, pp. 662-666.

[9] J. Hu, Y. Jin, and L. Dou, "A Time-based Cluster-Head Selection Algorithm for LEACH," IEEE Symposium on Computers and Communications, 2008, pp. 1172-1176.

[10] H. Yang and B. Sikdar, "Optimal Cluster Head Selection in the LEACH Architecture", IEEE International Conference on Performance, Computing, and Communications, 2007, pp. 93-100.

[11] D. Nam and H. Min, "An Energy-Efficient Clustering Using a Round-Robin Method in a Wireless Sensor Network".5th ACIS International Conference on Software Engineering Research, Management \& Applications, 2007, pp. 54-60.

[12] Security for Wireless Sensor Networks using Identity-Based Cryptography By Harsh Kupwade Patil, Stephen A. Szygenda (Section 7.2.1).Mobile Ad-hoc and Sensor Networks: First International Conference, MSN 2005 ...edited by Xiaohua Jia, Jie Wu, Yanxiang He (pp -918)

[13] I.F. Akyildiz et al., Wireless sensor networks: a survey, Computer Networks, vol. 38. no. 4, 2002, pp. 393-422.

[14] K. Sohrabi et al., Protocols for self-organization of a wireless sensor network, IEEE Personal Communications 7 (5) (2000) 1627

[15] R. Min et al., Low power wireless sensor networks, in: Proceedings of International Conference on VLSI Design, Bangalore, India, January 2001.

[16] F. Tariq, M. Rashid and Muhammad N. Khan, "Implementation of Smart Homes and Industrial Autmation System with Secure Communication over GSM," Universal Journal of Electrical and Electronic Engineering Vol. 3(4), pp. 125 - 131 DOI:10.13189/ujeee.2015.030403. 